\title{
Rare Case of a Malignant Proliferating Trichilemmal Tumor: A Case Report
}

\author{
Elias Fakhoury ${ }^{\mathrm{a}, \mathrm{d}}$, Anthony Cruz ${ }^{\mathrm{a}}$, Gary Kuehl ${ }^{\mathrm{b}}$, Matthew J. D’Alessio ${ }^{\mathrm{c}}$
}

\begin{abstract}
Proliferating Trichilemmal tumors (PTT) are rare neoplasms of the external root hair sheath that are largely benign. The potential for PTT to undergo transformation to a Malignant Proliferating Trichilemmal Tumor (MPTT) is unknown, with only about 40 cases of MPTT having ever been reported. We present an exceedingly rare case of a 22-year-old female who presented with an $8 \mathrm{~cm}$ mass of her anterior chest which was initially diagnosed as a squamous cell carcinoma and later classified as malignant proliferating trichilemmal tumor. In this case report we discuss the diagnosis, histo-pathological characteristics, and surgical treatment of such tumors.
\end{abstract}

Keywords: Malignant Proliferating Trichilemmal tumor; Proliferating Trichilemmal tumor; Squamous cell carcinoma; Pilar tumor; Epidermoid cyst

\section{Introduction}

Proliferating Trichilemmal tumors (PTT), also known as proliferating pilar tumors, are rare neoplasms of the external root hair sheath that are largely benign, cystic in nature, and characterized as containing trichilemmal keratin. These rare exophytic tumors are mainly confined to the scalp and back of the neck and most often reported in middle age females [1]. The potential for PTT to undergo transformation to a Malignant Proliferating Trichilemmal Tumor (MPTT) is un-

\footnotetext{
Manuscript accepted for publication November 5, 2012

${ }^{a}$ Nova Southeastern University College of Osteopathic Medicine, 2815

S. Seacrest Blvd., Boynton Beach Florida 33435, USA

${ }^{b}$ Pathology Department, Bethesda Memorial Hospital, 2815 S. Seacrest

Blvd., Boynton Beach Florida 33435, USA

${ }^{\mathrm{c}}$ Surgical Oncology, Bethesda Memorial Hospital, 2815 S. Seacrest

Blvd., Boynton Beach Florida 33435, USA

${ }^{\mathrm{d} C}$ Corresponding author: Elias Fakhoury, 2815 S. Seacrest Blvd.,

Boynton Beach Florida 33435, USA.

Email: fakhoury.elias@gmail.com
}

doi: http://dx.doi.org/10.4021/jmc1007e known, with only about 40 cases of MPTT having ever been reported. We present an exceedingly rare case of a 22 -yearold female with an $8.0 \mathrm{~cm}$ ulcerating mass of the anterior chest wall, which was initially diagnosed as a squamous cell carcinoma and later classified as malignant proliferating trichilemmal tumor.

\section{Case Report}

This 22-year-old Haitian-American female with no prior medical history, was referred to the Surgical Oncologist for evaluation of a large mass on her chest. The patient first noticed the mass 1 year prior with accelerated growth and ulceration occurring over the past 3 months. She also reported experiencing recent fevers, chills, and left neck and axillary swelling. Upon physical exam, an $8.0 \mathrm{~cm}$, exophytic, ulcerated mass was noted on her anterior chest, overlying the sternum. Shotty axillary lymphadenopathy was noted bilaterally. An incisional biopsy of the mass was taken in the office, and pathologic analysis was notable only for necrotic tissue with abscess formation, and was otherwise negative

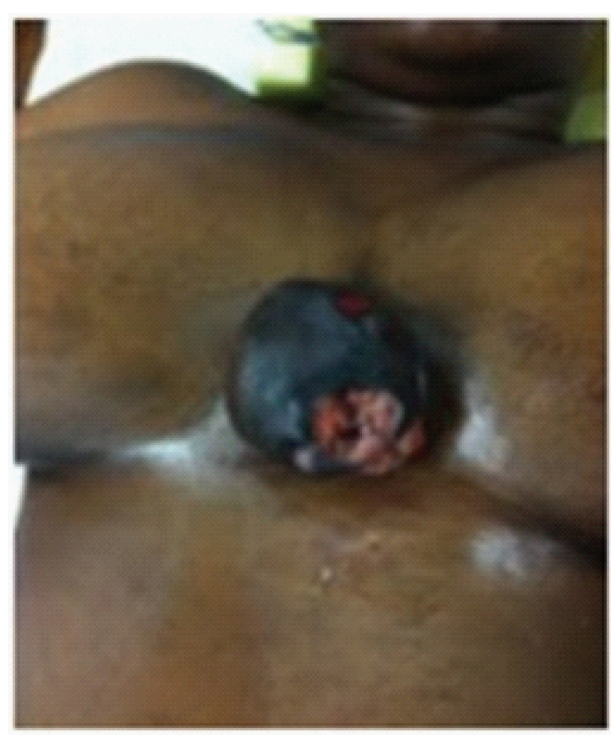

Figure 1. Gross view of $8.5 \mathrm{~cm}$ ulcerating mass. 


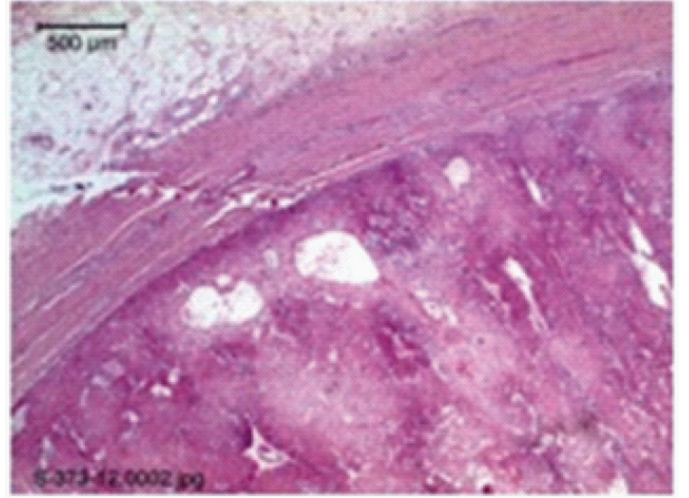

Figure 2. Trichilemmal tumor ulcerating and protruding from normal epidermis on the left, with the tumor on the right.

for a neoplastic process. Due to the high clinical suspicion for neoplasm, complete surgical excision of the mass was deemed necessary.

At the time of surgery, with the patient supine, the mass was noted on the anterior chest overlying the sternum and right pectoralis muscle. A wide excision of the tumor with 1 $\mathrm{cm}$ gross circumferential margins was performed. There was clinical evidence of neovascularity to the tumor. The pectoralis fascia was taken along with the tumor as a deep margin. The specimen underwent an intraoperative pathologic analysis with frozen section. Gross examination demonstrated a well-encapsulated tumor involving the skin and subcutaneous tissue, $8.5 \mathrm{~cm}$ in greatest dimension, and a weight of 215 grams (Fig. 1). There were two ulcerations $(2.5 \mathrm{~cm}$ and $3 \mathrm{~cm})$ and the margins were widely clear. Frozen section showed histologic abnormalities suggestive of squamous cell carcinoma.

Final reconstruction was deferred until the following day, as to obtain final margins as well as a lymphatic mapping for sentinel lymph node biopsy. The patient underwent bilateral axillary sentinel lymph node biopsies as indicated under the diagnosis of high-risk cutaneous squamous cell carcinoma. Pathologic analysis of the sentinel nodes demonstrated three benign lymph nodes with hyperplastic features; two were from the right axilla and one was from the left. Further cutaneous margins were taken circumferentially and revealed no histologic abnormalities.

Routine histopathologic analysis of the specimen demonstrated a tumor that was composed of a proliferation of squamous cells in which there were extensive areas of amorphous keratinization (Fig. 2). This histopathology is in line with follicular differentiation, and therefore the diagnosis of a proliferating trichilemmal tumor rather than a cutaneous squamous cell carcinoma. Furthermore due to the presence of small calcifications, numerous mitotic figures, apoptic bodies, marked cellular atypia, prominent necrosis, and an invasive growth pattern, the final diagnosis of a malignant proliferating trichilemmal tumor was made (Fig. 3).

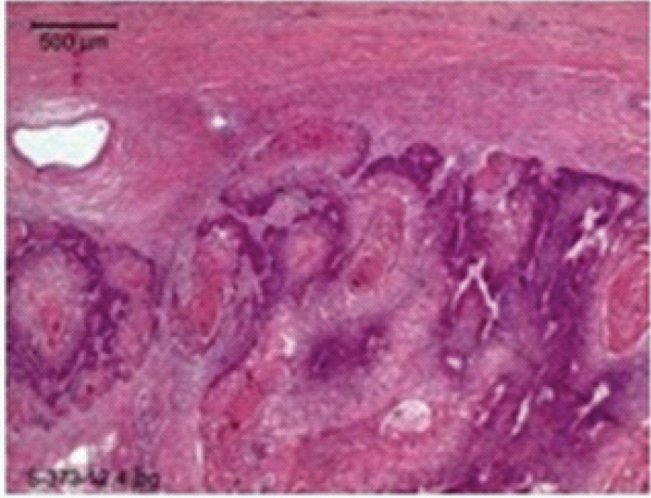

Figure 3. Infiltrative, poorly differentiated sheets and cords of tumor cells.

\section{Discussion}

Proliferating trichilemmal tumors (PTT) of the scalp are relatively uncommon. PTT of the chest is an even rarer condition that has been infrequently reported. These tumors were first named as proliferating epidermoid cysts by Wilson Jones in 1966 [2] and have been commonly misinterpreted both clinically and histologically as squamous cell carcinomas. They are five times more likely to occur in females and have a median age of occurrence of 65-year-old [3]. Although a predominantly benign condition, PTT has been reported to undergo a malignant transformation. Malignant proliferating trichilemmal tumor (MPTT) was first described by Saida et al in 1983 and is distinguished histologically by its infiltrative growth, cytological atypia, high mitotic activity, and lymph node metastases [4-6]. The potential for PTT to undergo malignant transformation is unknown and is an exceedingly rare occurrence.

Areas devoid of non-terminal hair, such as lanugo hair follicles of the bald scalp are unlikely to produce these tumors $[7,8]$. Pilar tumors are more common in areas with excess hair growth $[7,8]$ and less common in areas such as the anterior chest of a female as in our case or of the skin over the breast as reported by Uchida et al [9]. They gradually enlarge and the surface may breakdown and undergoes ulceration. Histological examination of malignant proliferating trichilemmal tumors reveal a well-circumscribed tumor composed of a proliferation of malignant - appearing squamous cells in which there is extensive areas of amorphous keratinization. The tumor cells overall are not well differentiated with mitotic figures scattered throughout and small areas of calcifications present. Grossly, MPTT usually presents as a mobile, firm, smooth and rounded nodule. Larger lesions may be lobular and multiple cysts are commonly found. Features which favored malignancy and a change of our initial diagnosis of squamous cell carcinoma included the large size, non-scalp location, infiltrative borders, nuclear pleomorphism, necrosis, rapid change in growth, and increased mitoses. 
The differential diagnoses of malignant proliferating trichilemmal tumors includes; squamous cell carcinoma, trichilemmal carcinoma, proliferating trichilemmal tumors, sebaceous cyst, and angiosarcoma. It is essential to properly diagnose due to the differences in the course and aggressiveness, the likelihood of recurrence and metastases, and the difference in recommended management of these differentials. Ye et al studied the clinicopathology of 76 cases in an attempt to classify such tumors and determine the clinical behavior of such trichilemmal tumors. They established certain histological criteria for these proliferating pilar tumors and stratified them into three groups ranging from benign to malignant. Our case is classified as a proliferating pilar tumor group 3. Ye et al define group 3 as exhibiting malignant behavior with invasive growth patterns, marked nuclear atypia, pathological mitotic forms, and geographic necrosis [7].

The current recommended management of malignant proliferating trichilemmal tumors is local excision with margins of normal tissue and a work-up to exclude metastases along with routine long-term follow-up [7]. Our case not only demonstrates the difficulty and importance of making a proper diagnosis, but also adds to the literature of these rare tumors. This unlikely patient, who does not follow the typical presentation of a malignant proliferating trichilemmal tumor as noted in previously reported cases, to our knowledge represents the youngest reported case of MPTT.

\section{References}

1. Holmes EJ. Tumors of lower hair sheath. Common histogenesis of certain so-called "sebaceous cysts," acanthomas and "sebaceous carcinomas". Cancer. 1968;21(2):234-248.

2. Jones EW. Proliferating epidermoid cysts. Arch Dermatol. 1966;94(1):11-19.

3. Carlin MC, Bailin PL, Bergfeld WF. Enlarging, painful scalp nodule. Proliferating trichilemmal tumor. Arch Dermatol. 1988;124(6):936, 938-939.

4. Trabelsi A, Stita W, Gharbi O, Kanani N, Sriha B, Korbi S. Malignant proliferating trichilemmal tumor of the scalp: a case report. Dermatol Online J. 2008;14(8):11.

5. Folpe AL, Reisenauer AK, Mentzel T, Rutten A, Solomon AR. Proliferating trichilemmal tumors: clinicopathologic evaluation is a guide to biologic behavior. J Cutan Pathol. 2003;30(8):492-498.

6. Ye J, Nappi O, Swanson PE, Patterson JW, Wick MR. Proliferating pilar tumors: a clinicopathologic study of 76 cases with a proposal for definition of benign and malignant variants. Am J Clin Pathol. 2004;122(4):566574.

7. Siddha M, Budrukkar A, Shet T, Deshpande M, Basu A, Patil N, Bhalavat R. Malignant pilar tumor of the scalp: a case report and review of literature. J Cancer Res Ther. 2007;3(4):240-243.

8. Saida T, Oohara K, Hori Y, Tsuchiya S. Development of a malignant proliferating trichilemmal cyst in a patient with multiple trichilemmal cysts. Dermatologica. 1983;166(4):203-208.

9. Uchida N, Tsuzuki Y, Ando T, Mochida Y, Yoshikawa M, Sekihara M, Kobayashi M, et al. Malignant proliferating trichilemmal tumor in the skin over the breast: a case report. Breast Cancer. 2000;7(1):79-82. 